\title{
History of the Cretan cohort of the Seven Countries Study
}

\author{
Christos M. Hatzis, Dimitra Sifaki-Pistolla, Anthony G. Kafatos
}

\begin{abstract}
Department of Social Medicine, Preventive Medicine and Nutrition Clinic, Medical School, University of Crete, Heraklion, Crete, Greece
\end{abstract}

\section{Dear Editors,}

It was a great pleasure to read the manuscript of Hatzis CM et al, 2013, published in the Hormones Journal. ${ }^{1}$ This article presented the results for cardiovascular risk factors of the Cretan cohort of the Seven Countries Study (SCS). The SCS was initiated in mid-1950 at the University of Minnesota by Professor Ancel Keys (Figure 1), with a follow-up

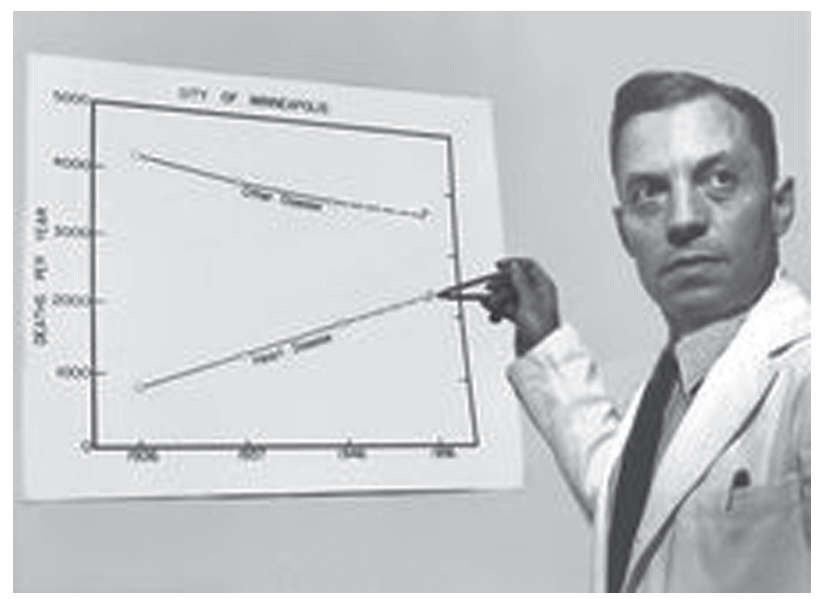

Figure 1. Professor Ancel Keys, University of Minnesota.

Key words: Cohort, Coronary heart disease, Crete, Seven Countries Study

Address for correspondence:

Dimitra Sifaki-Pistolla, MPH, PhDc, Voutes, University

Campus, P.O. Box: 2208, 71003 Iraklion, Crete, Greece,

Tel.: +30 2810394613 ,

E-mail: d.sifaki@med.uoc.gr, spdimi@gmail.com

Received 19-04-2015, Accepted 24-04-2015 every 10 years in most participating countries, the latest follow-up examination having been carried out in 2010. Keys recruited collaborators from seven countries, designing the first cross-cultural prospective study on coronary heart disease (CHD) in men pursuing different occupations and with different sociocultural backgrounds. ${ }^{2}$

The SCS is one of the longest multicultural cohorts and, for this reason, the presentation of its history and main findings is of major importance. It included 12,763 males aged between 40-59 years distributed over 16 cohorts among seven countries of four different parts of the world: the United States of America, Northern Europe, Southern Europe and Japan. The cohorts participating were USA (1 cohort), Finland (2 cohorts), the Netherlands (1 cohort), Italy (3 cohorts), Yugoslavia (5 cohorts), Greece ( 2 cohorts - Crete and Corfu) and Japan (2 cohorts) (Figure 2). The entry examination took place between 1958 and 1964 with $90 \%$ participation ranging from $75 \%$ in the USA and $100 \%$ in Japan.

Some of the main findings were that CHD risk at both the population and individual level was directly and independently related to the level of total serum cholesterol. CHD in the USA and Northern Europe greatly exceeded that of Southern Europe and persisted even when the population was controlled for age, serum cholesterol, blood pressure, smoking, physical activity and weight. East Finland had over 10 times higher mortality rates from CHD when compared with the Cretan rates, which showed the lowest CHD, cancer mortality and dementia in the 2000 follow-up..$^{3-5}$ These findings, in conjunction 


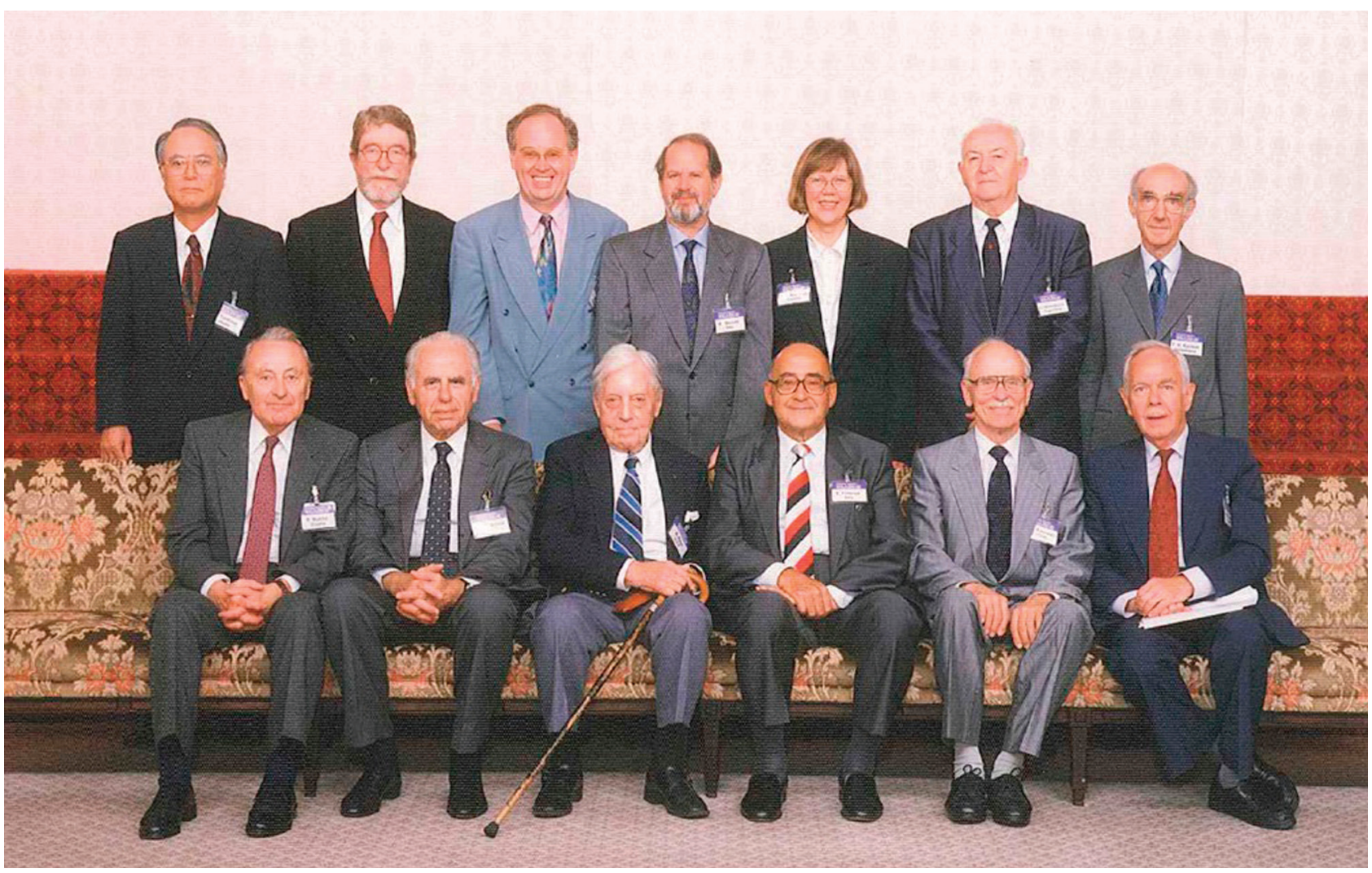

Figure 2. Representatives of 12 cohorts of the participant countries.

with the characteristic dietary habits of Crete, generated the term "Mediterranean diet". This study also demonstrated that the slowly changing habits of the Mediterranean populations, from a healthy lifestyle, physical activity and traditional dietary pattern to a less active lifestyle and eating habits influenced by western patterns of diet, increase risk for CHD significantly. ${ }^{6,7}$

The findings of the Cretan cohort were also of major importance. In 1989, the principal investigator, A. Keys (Figure 1), and the Greek original co-investigators, C. Aravanis and A. Dontas (Figures $3 \mathrm{~A}$ and $3 \mathrm{~B}$ ), were assigned to the Preventive Medicine and Nutrition Clinic of the Department of Social Medicine at the University of Crete to undertake the follow-up of the Cretan SCS cohort. The Preventive Medicine and Nutrition Clinic team designed the "1991 follow-up" of the total survivors from the 13 villages around the small town of Kastelli Pediados. The team, together with Professor A. Kafatos acting as the principal investigator of the team, visited all the villages and examined the 245 men who had survived amongst the original population of 686 men. The main findings indicated a significant increase (11.5\%) of total serum cholesterol between 1960 and 1991, while BMI and blood pressure were also significantly increased for all age groups. ${ }^{7}$ A 3-day weighted food record was obtained from a random sample of 21 men, which confirmed an increase in saturated fat intake and a decrease in monounsaturated fats over the past 31

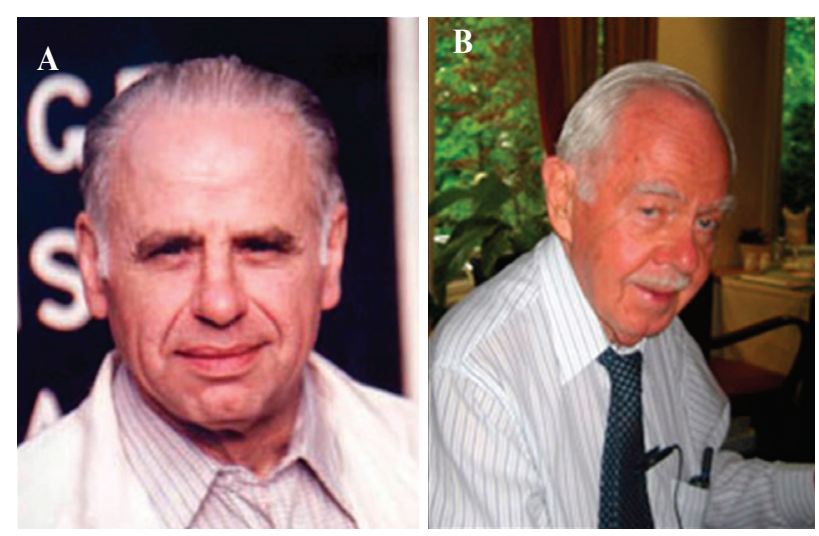

Figure 3. A. Greek original co-investigator, C. Aravanis. B. Greek original co-investigator, A. Dontas. 
years. Adipose fatty acid composition analysis also confirmed the above dietary changes by comparison with Christakis' study (a random population sample of 40-60 year-old men in Crete in 1962), which had analyzed adipose fat. ${ }^{8}$ Saturated fatty acid (SFA) in adipose tissue, which was $18.8 \%$ in 1962 , had increased to $19.5 \%$ in the SCS survivors in 2000 . This change in SFA of adipose tissue from 1962 to 2000 is attributable to high SFA intake from the diet and a parallel elevation of total serum cholesterol over a period of 38 years. By contrast, the Christakis study ${ }^{7}$ (1962) had found total serum cholesterol levels to be similar to those of the Keys study in 1960.

The most surprising findings of the SCS cohort in Crete is the low CHD, cancer and dementia mortality in Crete and the highest life expectancy in comparison to the other 15 cohorts of the study. These findings were mainly related to the dietary habits and lifestyle of men in Crete. Although there was a small increase in body weight of the SCS men in Crete over the 35 years of follow-up, it was significantly lower in comparison to all other European cohorts of the SCS. ${ }^{9}$ There was criticism of the Cretan cohort and it was refuted by the Rockefeller Foundation study in Crete in 1948. The criticism was that the population sample was biased, composed of a small number of farmers living in only one area of Crete and not representative of the rest of the island. Professor Albaugh designed a remarkable epidemiologic investigation by using multiple methods to define dietary intake in a representative sample of the population of Crete. ${ }^{10}$ The results on dietary intake among the population of Crete in those post-World War II years were strikingly similar to the SCS findings in 1960. Although this study lacked clinical nutrition and laboratory data, a later study in 1962 from Columbia University by Christakis reached results similar to those of the SCS in a random sample of 400 men from Crete. ${ }^{8}$

Another recent publication also supports the validity of the SCS Cretan cohort by demonstrating that the 50-year cancer mortality of this cohort is similar in variation and time trends to those of the entire island (common cancer mortality patterns). ${ }^{11}$ Another analysis of CHD (national mortality from 1960 to 2010) in Crete is presently being prepared which will be compared with the rest of Greece, with the Ionian Islands, where Corfu the second Greek
SCS cohort is located, and with the CHD national mortality data of the remaining six countries. A preliminary analysis demonstrates that the island of Crete has had the lowest CHD mortality for the last 50 years as the SCS cohort in Crete in comparison to the above regions. Another criticism of the SCS Cretan cohort was that the investigators had not evaluated the sugar intake, which is believed to be the most important factor regarding the etiology of atherosclerosis in comparison to SFA. It is true that the SCS publications do not include sugar intake. However, it is well known that for at least the first two decades from the initiation of SCS, the villages in Crete had no supermarkets and no processed foods with added sugar. Sugar consumption was limited occasionally to coffee and homemade sweets, while very often sugar was replaced by privately produced honey or petimezi syrup made from grapes. Crete and Corfu consumed less than $7 \mathrm{~kg}$ of sugar per person per year, while in Japan the quantity was $20 \mathrm{~kg}$ and $30 \mathrm{~kg}$ for England and Wales.

To conclude, the SCS Cretan cohort revealed the great benefits of the so-called Mediterranean diet, which differs considerably from the diet of the other Mediterranean countries (consumption of olive oil and fish, along with low consumption of meat). The very low meat intake in Crete was due to the fact that $60 \%$ of the men were strictly following the recommendation of the Orthodox Christian Church (OCC) by fasting for approximately 180 days/year. ${ }^{12}$ During that period, fish was more frequently consumed and sea food and snails were also consumed during the entire fasting period. A. Keys, the principal investigator of the SCS, underestimated this fact and was duly criticized, since the data on fasting could have provided valuable conclusions on morbidity, mortality and life expectancy during the 50-year follow-up period among those fasting and non-fasting. ${ }^{12}$

\section{REFERENCES}

1. Hatzis CM, Papandreou C, Patelarou E, et al, 2013 A 50-year follow-up of the Seven Countries Study: Prevalence of cardiovascular risk factors, food and nutrient intakes among Cretans. Hormones (Athens) 12: 379-385.

2. Keys A, 1970 Coronary heart disease in Seven Countries Study. Circulation 41: Suppl 1: 1-211.

3. Keys A, Aravanis C, Blackburn H, et al 1980 Seven 
Countries. A multivariate analysis of death and coronary heart disease. In: Cambridge, MA; Harvard University Press, pp: 1-381.

4. Keys A, Aravanis C, Blackburn H, et al, 1967 Epidemiological studies related to coronary heart disease. Characteristics of men aged 40-59 in Seven Countries. Acta Med Scand 460: Suppl 180: 1-392.

5. Alonso A, Jacobs DR Jr, Menotti A, et al, 2009 Cardiovascular risk factors and dementia mortality: 40 years of follow-up in the Seven Countries Study. J Neurol Sci 280: 79-83.

6. Kromhout D, Keys A, Aravanis C, et al, 1989 Food consumption patterns in the 1960s in Seven Countries Study. Am J Clin Nutr 49: 889-894.

7. Kafatos A, Diacatou A, Voukiklaris G, et al, 1997 Heart disease risk-factor status and dietary changes in the Cretan population over the past $30 \mathrm{y}$ : the Seven Countries Study. Am J Clin Nutr 65: 1882-1886.
8. Christakis G, Severinghaus E, Maldonado Z, Kafatos F, Hashim S, 1965 Crete: a study in the metabolic epidemiology of coronary heart disease. Am J Cardiol 15: 320-332.

9. Lanti M, Menotti A, Nedeljkovic S, Nissinen A, Kafatos A, Kromhout D, 2005 Long-term trends in major cardiovascular risk factors in cohorts of aging men in the European cohorts of the Seven Countries Study. Aging Clin Exp Res 17: 306-315.

10. Allbaugh LG 1953 Crete: a case study of an underdeveloped area. In: Princeton NJ: Princeton University Press.

11. Hatzis CM, Sifaki-Pistolla D, Papandreou C, Chlouverakis GI, Kafatos AG, Tzanakis NE, 2013 Validity of the cohort of Crete in the Seven Countries Study: A time-series study applied to the cancer mortality trend between 1960 and 2011. Oncol Lett 5: 964-968.

12. Cannon G, 2004 Out of the christmas box. Public Health Nutr 7: 987-990. 